\title{
Three-Dimensional Orthogonal Graph Drawing with Optimal Volume
}

\author{
Therese Biedl ${ }^{\star 1}$, Torsten Thiele ${ }^{2}$, and David R. Wood ${ }^{\star} 3$ \\ ${ }^{1}$ Department of Computer Science \\ University of Waterloo \\ Waterloo, ON N2L 3G1, Canada \\ biedl@uwaterloo.ca \\ ${ }^{2}$ Institut für Informatik \\ Freie Universität Berlin \\ Takustraße 19, 14195 Berlin, Germany \\ thiele@inf.fu-berlin.de \\ ${ }^{3}$ Basser Department of Computer Science \\ The University of Sydney \\ Sydney NSW 2006, Australia \\ davidw@cs . usyd.edu. au
}

\begin{abstract}
In this paper, we study three-dimensional orthogonal boxdrawings of graphs without loops. We provide lower bounds for three scenarios: (1) drawings where vertices have bounded aspect ratio, (2) drawings where the surface of vertices is proportional to their degree, and (3) drawings without any such restrictions. Then we give constructions that match the lower bounds in all scenarios within an order of magnitude.
\end{abstract}

\section{Introduction}

In this paper we consider three-dimensional orthogonal drawings of an $n$-vertex $m$-edge graph $G=(V, E)$ with maximum degree $\Delta$ (allowed to have parallel edges but no self loops). An orthogonal (box-)drawing of $G$ represents vertices by pairwise non-intersecting boxes in the three-dimensional grid. An edge $v w \in E$ is represented by a sequence of contiguous segments of grid lines possibly bent at grid points, between ports (points extremal in a particular direction) on the boxes of $v$ and $w$. Any two edge routes are disjoint except possibly at endpoints. The graph-theoretic terms 'vertex' and 'edge' will also refer to their representation in an orthogonal drawing. The number of ports on a box will be called its surface, and the number of grid points in a box is its volume. The aspect ratio of a box is its largest side length divided by its smallest side length.

\footnotetext{
* Research supported by NSERC.

** Supported by the Australian Research Council Large Grant A49906214, and partially completed while a PhD student in the School of Computer Science and Software Engineering at Monash University under the supervision of Dr Graham Farr.
}

J. Marks (Ed.): GD 2000, LNCS 1984, pp. 284295 2001.

(C) Springer-Verlag Berlin Heidelberg 2001 
An orthogonal drawing with a particular shape of box representing every vertex, e.g. point, line, or cube, is called an orthogonal shape-drawing for each particular shape. Orthogonal point-drawings have been studied in 78.91016 , 18. However, orthogonal point-drawings can only exist for graphs with maximum degree at most six. Overcoming this restriction has motivated recent interest in orthogonal box-drawings $351619 \mid 20$.

The smallest box enclosing an orthogonal drawing is called the bounding box. The bounding box volume and the maximum number of bends per edge are the most commonly proposed measures for determining the aesthetic quality of an orthogonal drawing. For box-drawings the size and shape of a vertex with respect to its degree are also considered an important measure of aesthetic quality.

An orthogonal drawing is said to be strictly $\alpha$-degree-restricted if for some constant $\alpha \geq 1$, the surface of $v$ is at most $\alpha \cdot \operatorname{deg}(v)$ for all vertices $v$; it is $\alpha$-degree-restricted if the surface of $v$ is at most $\alpha \cdot \operatorname{deg}(v)+o(\operatorname{deg}(v))$ for all $v$.

Lower Bounds: Let $\operatorname{vol}(G, r, \alpha)$ denote the minimum, taken over all orthogonal drawings of a graph $G$ that have aspect ratios at most $r$ and are strictly $\alpha$-degree restricted. Let $\operatorname{vol}(n, m, r, \alpha)$ be the maximum, taken over all graphs $G$ with $n$ vertices and $m$ edges, of $\operatorname{vol}(G, r, \alpha)$. Thus, $\operatorname{vol}(n, m, r, \alpha)$ describes a volume bound within which we can draw all graphs with $n$ vertices and $m$ edges such that each vertex $v$ has aspect ratio at most $r$ and surface at most $\alpha \cdot \operatorname{deg}(v)$.

The first lower bounds for 3-D orthogonal box-drawings were due to Hagihara $e t a l$. [12]. They show that, in the above notation, $\operatorname{vol}(n, m, 1,1)=\Omega(\max$ $\left.\left\{m n,(m / \log n)^{3 / 2}\right\}\right)$. In this paper, we show that

$-\operatorname{vol}(n, m, \infty, \infty)=\Omega(m \sqrt{n})$,

$-\operatorname{vol}(n, m, r, \infty)=\Omega\left(m^{3 / 2} / \sqrt{r}\right)$,

$-\operatorname{vol}(n, m, \infty, \alpha)=\Omega\left(m^{3 / 2} / \alpha\right)$.

We thus improve the results of [12] in three ways: Firstly, we remove the $\log$-factor, to establish $\operatorname{vol}(n, m, 1,1)=\Omega\left(m^{3 / 2}\right)$ as the lower bound. Secondly, we prove this result even if only one of having bounded aspect ratios and being strictly degree-restricted holds. Finally, we also study the case when neither of these two conditions hold, and establish a weaker, but optimal, lower bound. Our first result includes the lower bound of $\Omega\left(n^{5 / 2}\right)$ for orthogonal drawings of $K_{n}$ established by Biedl et al. [5]. In fact, the proof of our lower bounds are based on techniques developed in this paper, though we generalize them to graphs with an arbitrary number of edges and include considerations of bounded aspect ratios and degree restriction.

Algorithms: A trade-off between the maximum number of bends per edge route and the bounding box volume is apparent in algorithms for orthogonal graph drawing; see Table 1 for an overview of the known results.

In this paper, we present two optimal algorithms for orthogonal graph drawing. The first algorithm produces degree-restricted orthogonal cube-drawings with $\mathcal{O}\left(\mathrm{m}^{3 / 2}\right)$ bounding box volume and at most six bends per edge. The technique used is a generalization of the COMPACT algorithm of Eades et al. [10] 
Table 1. The tradeoff between bounding box volume and the maximum number of bends in orthogonal graph drawings. All lower bounds are proved in Theorem 2.

\begin{tabular}{|c|c|c|c|c|c|}
\hline lower bound & volume & bends & model & graphs & reference \\
\hline \multicolumn{6}{|c|}{ bounded aspect ratio / degree-restricted } \\
\hline$\Omega\left(m^{3 / 2}\right)$ & $\mathcal{O}\left((n m)^{3 / 2}\right)$ & 2 & general position & simple & \begin{tabular}{l|l|l|}
3 & 19 \\
\end{tabular} \\
\hline$\Omega\left(m^{3 / 2}\right)$ & $\mathcal{O}(n m \sqrt{\Delta})$ & 2 & lifting $\frac{1}{2}$-edges & simple & 3 \\
\hline$\Omega\left(m^{3 / 2}\right)$ & $\mathcal{O}\left(m^{2}\right)$ & 5 & plane layout & multigraphs & Thm. 目 \\
\hline$\Omega\left(m^{3 / 2}\right)$ & $\mathcal{O}\left((n \Delta)^{3 / 2}\right)$ & $10^{1}$ & plane layout & simple & 12 \\
\hline$\Omega\left(m^{3 / 2}\right)$ & $\mathcal{O}\left(m^{3 / 2}\right)$ & 6 & plane layout & multigraphs & Thm. 3 \\
\hline \multicolumn{6}{|c|}{ no bounds on aspect ratio / degree-restricted } \\
\hline$\Omega\left(m^{3 / 2}\right)$ & $\mathcal{O}\left(n^{2} m\right)$ & 2 & general position & simple & 319 \\
\hline$\Omega\left(m^{3 / 2}\right)$ & $\mathcal{O}\left(n^{2} \Delta\right)$ & 2 & lifting $\frac{1}{2}$-edges & simple & 3 \\
\hline$\Omega\left(m^{3 / 2}\right)$ & $\mathcal{O}\left(m^{2}\right)$ & 5 & plane layout & multigraphs & Thm. 4 \\
\hline$\Omega\left(m^{3 / 2}\right)$ & $\mathcal{O}\left(m^{3 / 2}\right)$ & 6 & plane layout & multigraphs & Thm. 3 \\
\hline \multicolumn{6}{|c|}{ no bounds on aspect ratio / not necessarily degree-restricted } \\
\hline$\Omega(m \sqrt{n})$ & $\mathcal{O}\left(n^{3}\right)$ & 1 & lifting edges & simple & 5 \\
\hline$\Omega(m \sqrt{n})$ & $\mathcal{O}\left(n^{5 / 2}\right)$ & 3 & lifting edges & simple & 5 \\
\hline & $\mathcal{O}(m n)$ & 3 & & multigr & Thm. 6 \\
\hline$\Omega(m \sqrt{n})$ & $\mathcal{O}(m \sqrt{n})$ & 4 & plane layout & simple & Thm. 5 \\
\hline
\end{tabular}

for orthogonal point-drawing, and is an improvement on the algorithms of Hagihara et al. [12] and Wood [20], who obtained upper bounds of $\mathcal{O}\left((n \Delta)^{3 / 2}\right)$ and $\mathcal{O}\left(m^{2} / \sqrt{n}\right)$, respectively. Our second algorithm produces orthogonal rectangledrawings with $\mathcal{O}(m \sqrt{n})$ bounding box volume and at most four bends per edge; the drawings are not necessarily degree-restricted nor do the vertices have bounded aspect ratios. Both upper bounds are therefore within an order of magnitude of the lower bound. We also present refinements of both our algorithms with one less bend per edge, at the cost of an increase in the volume.

\section{Lower Bounds}

In this section we prove lower bounds using graphs that have $\Omega(m)$ edges in any cut with at least $n / 6$ vertices on each side of the cut.

Lemma 1. If $p \neq q$ are primes, $p \equiv 1 \bmod 4, q \equiv 1 \bmod 4,144 \leq p<q(q-$ $1) / 2$, then there exists a simple graph $G_{p, q}$ with the following properties:

- $G_{p, q}$ is d-regular for $d=p+1$,

- the number $n$ of vertices of $G_{p, q}$ is at least $q(q-1) / 2$ and at most $q(q-1)$.

- for any disjoint sets $S, T \subset V\left(G_{p, q}\right)$ with $|S||T| \geq n^{2} / 36$ there are at least $C \cdot d n$ edges between $S$ and $T$, where $C>0.00009$ is a constant.

${ }^{1}$ Hagihara et al. 12 did not count the number of bends per edge; we deduce the bound of 10 from their construction. 
Proof. (Sketch) Let $G_{p, q}$ be the Ramanujan graph $X^{p, q}$ defined in [15]; the first two properties of the graph were shown in this paper. It was also shown that $\lambda \leq$ $2 \sqrt{d-1}$, where $\lambda$ denotes the second-largest eigenvalue of $G_{p, q}$. Assume $S$ and $T$ are disjoint vertex sets with $|S||T| \geq n^{2} / 36$. We know from [2] that the number of edges between $S$ and $T$ is at least $\frac{\overline{d|S||T|}}{n}-\lambda \sqrt{|S||T|}$. Using $6 \sqrt{|S||T|} / n \geq 1$ and $\sqrt{d} / \sqrt{145} \geq 1$, one can show that $\lambda \sqrt{|S||T|} \leq 12 / \sqrt{145} \cdot d|S||T| / n$, and hence the number of edges between $S$ and $T$ is at least $(1-12 / \sqrt{145}) \cdot d|S||T| / n \geq$ $\frac{1}{36}(1-12 / \sqrt{145}) d n$.

Our lower bound proof is based on the technique developed in [5], which distinguishes three cases: either many vertices are intersected by one grid line, or many vertices are intersected by one plane, or neither of these is the case.

Theorem 1. Let $G=(V, E)$ be an $n$-vertex d-regular simple graph $(n \geq 8)$ such that for any disjoint sets $V_{1}, V_{2} \subset V$ with $\left|V_{1}\right|\left|V_{2}\right| \geq n^{2} / 36$ there are at least $C \cdot d n$ edges, $0<C \leq 1$ between $V_{1}$ and $V_{2}$. Then

$$
\begin{aligned}
& -\operatorname{vol}(G, \infty, \infty) \geq \frac{1}{3} C^{3 / 2} \cdot d n^{3 / 2} . \\
& -\operatorname{vol}(G, r, \infty) \geq \frac{1}{3} C^{3 / 2} \cdot(d n)^{3 / 2} / \sqrt{r}, r \geq 1 . \\
& -\operatorname{vol}(G, \infty, \alpha) \geq \frac{1}{3} C^{2} \cdot(d n)^{3 / 2} / \alpha, \alpha \geq 1
\end{aligned}
$$

Proof. Consider a drawing of $G$ in a grid of dimensions $X \times Y \times Z$. For ease of proof we assume that $n$ is divisible by 6 ; see $[6$ for a proof without this assumption.

Case 1: A line intersects many vertices. Assume that a $Z$-line (i.e., a gridline parallel to the $Z$-axis) intersects at least $\frac{1}{3} n$ vertices. Let $Z_{0}$ be such that the $\left(Z=Z_{0}\right)$-plane intersects none of these vertices and separates them into two groups of at least $\frac{1}{6} n$ vertices each. By assumption at least $C \cdot d n$ edges connect these two groups. These edges cross the $\left(Z=Z_{0}\right)$-plane, which thus must contain at least $C \cdot d n$ points with integer $X$ - and $Y$-coordinates. Hence $X Y \geq C \cdot d n$. Since the $Z$-line intersects at least $\frac{1}{3} n$ vertices, we have $Z \geq \frac{1}{3} n$, so $X Y Z Z \geq \frac{1}{3} C \cdot d n^{2}$, which proves all claims by $C \leq 1$ and $d \leq n$.

Case 2: No plane intersects many vertices. Assume that any $X$-plane, $Y$ plane or $Z$-plane (i.e., a plane perpendicular to the $X$-axis, $Y$-axis or $Z$-axis, respectively) intersects at most $\frac{2}{3} n$ vertices. A vertex is left of an $\left(X=X_{0}\right)$-plane if all the points in its grid box have $X$-coordinates less than $X_{0}$. The notion of right of an $\left(X=X_{0}\right)$-plane is analogous. Let $X^{\prime}$ be the largest integral value such that fewer than $\frac{1}{6} n$ vertices are left of the $\left(X=X^{\prime}\right)$-plane. Since the $\left(X=X^{\prime}\right)$ plane intersects at most $\frac{2}{3} n$ vertices, there are at least $\frac{1}{6} n$ vertices right of the $\left(X=X^{\prime}\right)$-plane. All these vertices also lie to the right of $\left(X=X^{\prime}+\frac{1}{2}\right)$-plane. By definition of $X^{\prime}$, at least $\frac{1}{6} n$ vertices lie left of the $\left(X=X^{\prime}+1\right)$-plane. All these vertices also lie to the left of $\left(X=X^{\prime}+\frac{1}{2}\right)$-plane.

By assumption there are at least $C \cdot d n$ edges between the vertices on the left and the vertices on the right of the $\left(X=X^{\prime}+\frac{1}{2}\right)$-plane, so $Y Z \geq C \cdot d n$. Since the same argument holds for the other two directions, $X Y Z=\sqrt{X Y \cdot Y Z \cdot X Z} \geq$ $(C \cdot d n)^{3 / 2}$, which proves all claims. 
Case 3: Neither of the above. Assume now that any $X$-line, $Y$-line or $Z$ line intersects at most $\frac{1}{3} n$ vertices, but there exists, say, a $\left(Z=Z_{0}\right)$-plane that intersects at least $\frac{2}{3} n$ vertices. As an $\left(X=X_{0}\right)$-plane is swept from smaller to larger values of $X_{0}$, the $Y$-line determined by the intersection of this $\left(X=X_{0}\right)$ plane with the $\left(Z=Z_{0}\right)$-plane sweeps the $\left(Z=Z_{0}\right)$-plane. At any time, this $Y$-line intersects at most $\frac{1}{3} n$ vertices by assumption. Similarly as above, one can therefore find a $\left(X=X_{0}\right)$-plane that splits the vertices intersected by the $\left(Z=Z_{0}\right)$-plane into two sets $V_{-}$and $V_{+}$to the left and right of it which each contain at least $\frac{1}{6} n$ vertices. By assumption there are at least $C \cdot d n$ edges between $V_{-}$and $V_{+}$, so $Y Z \geq C \cdot d n$. Apply exactly the same argument in the $Y$-direction to obtain $X Z \geq C \cdot d n$. We get the three lower bounds as follows:

- The $\left(Z=Z_{0}\right)$-plane intersects at least $\frac{2}{3} n$ vertices, so $X Y \geq \frac{2}{3} n$. This implies $X Y Z=\sqrt{X Y \cdot Y Z \cdot X Z} \geq \sqrt{\frac{2}{3} n \cdot(C \cdot d n)^{2}}=\sqrt{\frac{2}{3}} C \cdot d n^{3 / 2}$, which proves the first lower bound.

- Assume that every vertex has aspect ratio at most $r(r \geq 1)$. In particular therefore, $Z(v) \leq r X(v)$ and $Z(v) \leq r Y(v)$ for every vertex $v$ represented by an $X(v) \times Y(v) \times Z(v)$-box. Since the surface of $v$ is at least $\operatorname{deg}(v)$, this implies $X(v) Y(v) \geq \operatorname{deg}(v) / 6 r=d / 6 r$. Since the $\left(Z=Z_{0}\right)$-plane intersects at least $\frac{2}{3} n$ vertices, and these intersections are disjoint, there must be at least $\frac{2}{3} n \cdot d / 6 r$ integral points in the $\left(Z=Z_{0}\right)$-plane. So $X Y \geq \frac{1}{9} \cdot d n / r$ and $X Y Z=\sqrt{X Y \cdot Y Z \cdot X Z} \geq \sqrt{\frac{1}{9} \cdot d n / r \cdot(C \cdot d n)^{2}}=\frac{1}{3} C \cdot(d n)^{3 / 2} / \sqrt{r}$, which proves the second lower bound.

- Assume that the surface of every vertex $v$ is at most $\alpha \operatorname{deg}(v)=\alpha d(\alpha \geq 1)$, which implies $Z(v)<\alpha d / 4$. Define $Z_{-}=Z_{0}-\alpha d / 4$ and $Z_{+}=Z_{0}+\alpha d / 4$. We say that a point is inside if its $Z$-coordinate $z$ satisfies $Z_{-}<z<Z_{+}$, and outside otherwise. Note that all vertices in $V_{-}$and $V_{+}$cross the $\left(Z=Z_{0}\right)$ plane, hence they can cross neither the $\left(Z=Z_{-}\right)$-plane nor the $\left(Z=Z_{+}\right)$plane, and all grid points of all vertices in $V_{-}$and $V_{+}$are inside.

Recall that the $\left(X=X_{0}\right)$-plane separates the vertices in $V_{+}$and $V_{-}$and hence is crossed by at least $C \cdot d n$ edges. There are only $Y \cdot \alpha d / 2$ integral points that are inside and on the $\left(X=X_{0}\right)$-plane, so at least $C \cdot d n-Y \cdot \alpha d / 2$ edges cross the $\left(X=X_{0}\right)$-plane at an outside point.

Each of these $C \cdot d n-Y \cdot \alpha d / 2$ edges starts at a vertex in $V_{-}$at an inside point, crosses the $\left(X=X_{0}\right)$-plane at an outside point, and ends at a vertex in $V_{+}$ at an inside point. Hence each such edge crosses the $\left(Z=Z_{-}\right)$-plane or the $\left(Z=Z_{+}\right)$-plane at least twice. These two planes together therefore must have at least $2(C \cdot d n-Y \cdot \alpha d / 2)$ grid points, therefore $X Y \geq C \cdot d n-Y \cdot \alpha d / 2$. Applying the exact same argument in the $Y$-direction, we obtain $X Y \geq$ $C \cdot d n-X \cdot \alpha d / 2$.

Now, if $X \leq C n / \alpha$ or $Y \leq C n / \alpha$ then $X Y \geq C \cdot d n / 2$, and therefore $X Y Z=$ $\sqrt{X Y \cdot Y Z \cdot X Z} \geq \sqrt{\frac{1}{2} C \cdot d n \cdot(C \cdot d n)^{2}}=\frac{1}{\sqrt{2}} C^{3 / 2} \cdot(d n)^{3 / 2}$. If $X>C n / \alpha$ and $Y>C n / \alpha$, then $X Y>(C n)^{2} / \alpha^{2}$, and $X Y Z=\sqrt{X Y \cdot Y Z \cdot X Z} \geq$ $\sqrt{\left(C^{2} \cdot n^{2} / \alpha^{2}\right) \cdot(C \cdot d n)^{2}}=C^{2} \cdot d n^{2} / \alpha \geq C^{2} \cdot(d n)^{3 / 2} / \alpha$ by $d \leq n$. Either way, the third claim is proved. 
Now we extend the result to arbitrary values of $m$ and $n$, as long as both are big enough.

Lemma 2. There exists constants $x_{1}$ and $k \geq 2$ such that for all $x \geq x_{1}$, the interval $\left[\frac{1}{k} x, x\right]$ contains a prime number $p$ with $p \equiv 1 \bmod 4$.

Proof. (Sketch) This follows from a famous theorem by de la Vallée Poussin that establishes that the number of primes $p \leq x$ with $p \equiv 1 \bmod 4$ is proportional to $x / \log x$ (see e.g. [1] for a proof).

Lemma 3. For any sufficiently large $n$, and any sufficiently large $m \leq\left(\begin{array}{c}n \\ 2\end{array}\right)$, there exists a simple graph $G$ with $n$ vertices and $m$ edges that has a $d^{\prime}$-regular subgraph $G^{\prime}$ with $n^{\prime}$ vertices and $m^{\prime}$ edges such that

- $G^{\prime}$ satisfies the conditions of Theorem 1 with constant $C^{\prime} \geq C$,

$-n \geq n^{\prime} \geq n / 8 k^{2}$

$-m \geq m^{\prime}=d^{\prime} n^{\prime} / 2 \geq m / 64 k^{4}$,

- any vertex of $G^{\prime}$ has degree $\leq 8 k^{2} d^{\prime}$ in $G$,

where $k \geq 2$ is the constant of Lemma国.

Proof. (Sketch) Assume $n \geq \max \left\{32 k^{4}, 2 x_{1}^{2}\right\}$ and $m \geq \max \left\{\frac{1}{4}\left(x_{1}+1\right) n, \frac{145}{4} k n\right\}$, where $x_{1}$ is the lower bound on $x$ in Lemma 2. The proof splits into two cases, depending on whether $m$ is big or not.

If $m \geq n^{2} / 32 k^{4}$, then let $G^{\prime}$ be the complete graph on $n^{\prime}=\left\lceil n / 8 k^{2}\right\rceil+1$ vertices. To obtain $G$, add $n-n^{\prime}$ additional vertices and $m-\left(\begin{array}{c}n^{\prime} \\ 2\end{array}\right)$ arbitrary edges such that the resulting graph is simple. Using the assumptions on $n$ and $m$ one can verify all conditions.

If $m<n^{2} / 32 k^{4}$, then let $q^{\prime}=\frac{1}{2}+\sqrt{\frac{n}{2}+\frac{1}{4}}$, and find a prime $q$ with $q \equiv$ $1 \bmod 4$ such that $\frac{1}{k} q^{\prime} \leq q \leq q^{\prime}$. Let $p^{\prime}=2 m / q(q-1)-1$ and find a prime $p$ with $p \equiv 1 \bmod 4$ such that $\frac{1}{k} p^{\prime} \leq p \leq p^{\prime}$. Using the conditions on $n$ and $m$ one verifies $q^{\prime}, p^{\prime} \geq x_{1}$, so this is possible. Also, $p \neq q, p \geq 144$ and $p<q(q-1) / 2$. Let $G^{\prime}$ be the graph $G_{p, q}$ as in Lemma 1 and suppose it has $n^{\prime}$ vertices and $m^{\prime}$ edges. Create $G$ by adding $n-n^{\prime}$ vertices and $m-m^{\prime}$ edges between them; by $m<n^{2} / 32 k^{4}$ one can show $m-m^{\prime} \leq\left(\begin{array}{c}n-n^{\prime} \\ 2\end{array}\right)$ and this can be done such that $G$ is simple and the degrees in $G^{\prime}$ are unchanged. Using the assumptions on $n$ and $m$ one can verify all conditions.

Theorem 2. $\operatorname{vol}(n, m, \infty, \infty)=\Omega(m \sqrt{n}), \operatorname{vol}(n, m, r, \infty)=\Omega\left(m^{3 / 2} / \sqrt{r}\right)$, and $\operatorname{vol}(n, m, \infty, \alpha)=\Omega\left(m^{3 / 2} / \alpha\right)$.

Proof. (Sketch) Let $G$ be the graph of Lemma 3. Any drawing of $G$ contains a drawing of $G^{\prime}$, and thus has volume $\geq \frac{1}{3} C^{\prime 3 / 2} d^{\prime}\left(n^{\prime}\right)^{3 / 2}$ by Theorem 1 . If the drawing of $G$ has aspect ratios bounded by $r$, then so does the drawing of $G^{\prime}$, which thus has volume $\geq \frac{1}{3} C^{\prime 3 / 2}\left(d^{\prime} n^{\prime}\right)^{3 / 2} / \sqrt{r}$. If the drawing of $G$ is strictly $\alpha$-degree restricted, then every vertex in $G^{\prime}$ has surface $\leq \alpha 8 k^{2} d^{\prime}$. Thus the drawing of $G^{\prime}$ is strictly $\left(\alpha 8 k^{2}\right)$-degree restricted, and has volume at $\geq \frac{1}{3} C^{\prime 2}\left(d^{\prime} n^{\prime}\right)^{3 / 2} / \alpha 8 k^{2}$. Using the bounds on $C^{\prime}, n^{\prime}$ and $m^{\prime}$ known from Lemma 3, one obtains the results since $k$ is a constant. 


\section{Constructions}

In the following, we give two constructions. The first one creates cube-drawings with asymptotically optimal volume, the second one creates drawings without restrictions on vertex boxes that have asymptotically optimal volume.

\subsection{Cube-Drawings}

In the following algorithm for producing orthogonal drawings, vertices are initially represented by squares in the $(Z=0)$-plane, and edges are routed above the plane. Vertices are then extended in the $Z$-dimension to form cubes. For space reasons, we give here only a simplified construction with larger constants.

1. Represent each vertex $v \in V$ by a square $S_{v}$ of sidelength $2\lceil\sqrt{\operatorname{deg}(v)}\rceil+2.2$

2. Position the squares $\left\{S_{v}: v \in V\right\}$ in the $(Z=0)$-plane with the squarepacking algorithm of Kleitman and Krieger [13.

3. For each vertex $v \in V$, remove the top two rows from $S_{v}$ and the two rightmost columns from $S_{v}$. Vertices are now disjoint; see Fig. 1).

4. Direct edges arbitrarily, and assign each edge $v w \in E$ unique $Z^{+}$ports (ports extreme in the $Z^{+}$direction) at $v$ and $w$ both with even $X$-coordinate and even $Y$-coordinate.

5. Construct a graph $H$ with $V(H)=E$. For oriented edges $v w, x y \in E$, add the edge $\{v w, x y\}$ to $E(H)$ if the ports of $v w$ at $v$ and of $x y$ at $x$ are in the same column, or if the ports of $v w$ at $w$ and of $x y$ at $y$ are in the same row.

6. Vertex colour the graph $H$ with $\Delta(H)+1$ colours, and for each vertex $v \in V(H)$ coloured $i$ corresponding to an edge $v w$, set $h(v w) \leftarrow i$.

7. For each oriented edge $v w \in E$ construct an edge route for $v w$ as follows. Suppose the ports on $v$ and $w$ assigned to $v w$ have coordinates $\left(v_{X}, v_{Y}, 0\right)$ and $\left(w_{X}, w_{Y}, 0\right)$, respectively. Route the edge $v w$ with one of the following four or six bend routes, as illustrated in Fig. 1.

$$
\begin{aligned}
- & v_{X}=w_{X}: \\
& \left(v_{X}, v_{Y}, 0\right) \rightarrow\left(v_{X}, v_{Y}, 2 h(v w)\right) \rightarrow\left(v_{X}+1, v_{Y}, 2 h(v w)\right) \rightarrow \\
& \left(v_{X}+1, w_{Y}, 2 h(v w)\right) \rightarrow\left(v_{X}, w_{Y}, 2 h(v w)\right) \rightarrow\left(v_{X}, w_{Y}, 0\right) \\
- & v_{Y}=w_{Y}: \\
& \left(v_{X}, v_{Y}, 0\right) \rightarrow\left(v_{X}, v_{Y}, 2 h(v w)+1\right) \rightarrow\left(v_{X}, v_{Y}+1,2 h(v w)+1\right) \rightarrow \\
& \left(w_{X}, v_{Y}+1,2 h(v w)+1\right) \rightarrow\left(w_{X}, v_{Y}, 2 h(v w)+1\right) \rightarrow\left(w_{X}, v_{Y}, 0\right) \\
- & v_{X} \neq w_{X} \text { and } v_{Y} \neq w_{Y}: \\
& \left(v_{X}, v_{Y}, 0\right) \rightarrow\left(v_{X}, v_{Y}, 2 h(v w)\right) \rightarrow\left(v_{X}+1, v_{Y}, 2 h(v w)\right) \rightarrow \\
& \left(v_{X}+1, w_{Y}+1,2 h(v w)\right) \rightarrow\left(v_{X}+1, w_{Y}+1,2 h(v w)+1\right) \rightarrow \\
& \left(w_{X}, w_{Y}+1,2 h(v w)+1\right) \rightarrow\left(w_{X}, w_{Y}, 2 h(v w)+1\right) \rightarrow\left(w_{X}, w_{Y}, 0\right)
\end{aligned}
$$

8. Enlarge squares representing vertices into cubes by extending their side parallel to the $Z$-axis.

${ }^{2}$ The algorithm by Hagihara et al. [12] is similar in spirit, but uses a square of size $\mathcal{O}(\sqrt{\Delta})$ for each vertex, hence resulting in an $\mathcal{O}\left((n \Delta)^{3 / 2}\right)$ volume drawing. 


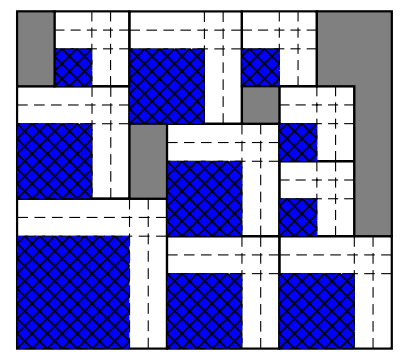

(a)

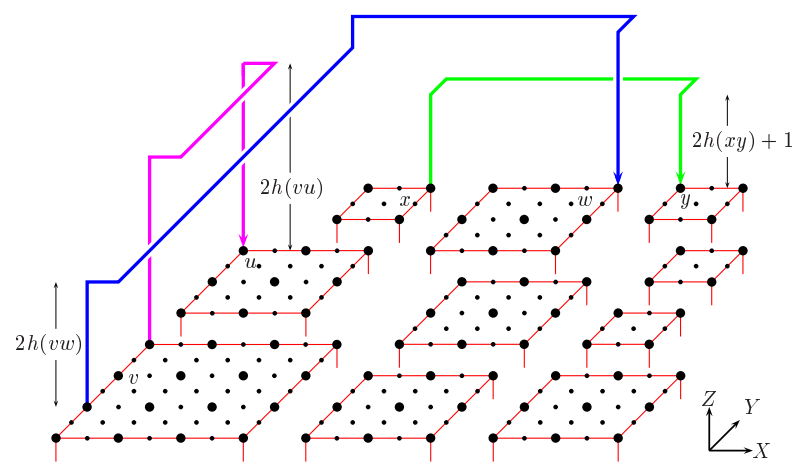

(b)

Fig. 1. (a) Square packing, (b) Routing edges.

Theorem 3. The above algorithm determines a 24-degree-restricted orthogonal cube-drawing of any loopless graph $G$ in $\mathcal{O}\left(\mathrm{m}^{3 / 2}\right)$ time, with $\mathcal{O}\left(\mathrm{m}^{3 / 2}\right)$ bounding box volume (assuming $m=\Omega(n)$ ) and at most six bends per edge route.

Proof. (Sketch) The squares $\left\{S_{v}: v \in V\right\}$ have area $\sum_{v}(2\lceil\sqrt{\operatorname{deg}(v)}\rceil+2)^{2}=$ $8 m+o(m)$. The algorithm of [13] packs squares with a total area of 1 in a $2 / \sqrt{3} \times \sqrt{2}$ rectangle. So the squares $\left\{S_{v}: v \in V(G)\right\}$ can be packed in a rectangle with size $(2 \sqrt{8 m} / \sqrt{3}+o(\sqrt{m})) \times(\sqrt{8 m} \cdot \sqrt{2}+o(\sqrt{m}))=\mathcal{O}(\sqrt{m}) \times \mathcal{O}(\sqrt{m})$. Hence the maximum degree of $H$ is $\mathcal{O}(\sqrt{m})$, the height of the drawing above the $(Z=0)$-plane is also $\mathcal{O}(\sqrt{m})$. Expanding vertices adds height $\leq 2\lceil\sqrt{\Delta}\rceil \leq \sqrt{m}$. Hence the total height is $\mathcal{O}(\sqrt{m})$, which proves the bound on the volume.

The surface of a vertex $v$ is $6(2\lceil\sqrt{\operatorname{deg}(v)}\rceil)^{2}=24 \operatorname{deg}(v)+o(\operatorname{deg}(v))$. By construction, there are at most six bends per edge route.

The time-consuming stage of the algorithm is the vertex colouring of $H$, which takes $\mathcal{O}(|E(H)|)=\mathcal{O}(|V(H)| \Delta(H))=\mathcal{O}(m \sqrt{m})=\mathcal{O}\left(m^{3 / 2}\right)$ time.

By routing the edges above and below the vertices, 12-degree-restricted vertices are possible; see [6] for details. Very recently, Biedl and Chan [4] developed a technique to implement steps 5 and 6 of our algorithm more efficiently. With this technique, the time complexity of our algorithm reduces to $\mathcal{O}(m \log m)$, and the volume decreases to $\approx 83 m^{3 / 2}+o\left(m^{3 / 2}\right)$.

If we remove the middle segment from each 6 -bend edge route and route each edge with unique height, then the overall height is $\mathcal{O}(m)$ and we obtain the following result.

Theorem 4. Every loopless graph has a 12-degree-restricted orthogonal cubedrawing, which can be computed in $\mathcal{O}(m)$ time, with $\mathcal{O}\left(m^{2}\right)$ bounding box volume and at most five bends per edge route. 


\subsection{Drawings with Unbounded Aspect Ratio}

We now show how to create orthogonal drawings of simple graphs that have volume $\mathcal{O}(m \sqrt{n})$; the vertices have unbounded aspect ratios and are not necessarily degree-restricted; Vertices are initially represented by points or line segments in the $(Z=0)$-plane. The vertices are then extended in the $Z$-dimension to form lines or rectangles. Let $N=\lceil\sqrt{n}\rceil$.

1. Define $V_{\text {big }}=\{v \in V: \operatorname{deg}(v) \geq 4 m / N\}, V_{\text {small }}=V-V_{\text {big }}$. Define $E_{\text {big }}$ to be the set of edges with both endpoints in $V_{b i g}, E_{c u t}$ to be the set of edges with exactly one endpoint in $V_{b i g}$, and $E_{\text {small }}$ to be the remaining edges.

2. For each vertex $v \in V_{\text {small }}$, represent $v$ by a $2 \times 2$-rectangle $S_{v}$.

3. For each vertex $v \in V_{\text {big }}$, define $k(v)=\lceil\operatorname{deg}(v) N / 4 m\rceil$, and represent $v$ by a $2 k(v) \times 2$-rectangle $S_{v}$.

4. Let $w_{1}, \ldots, w_{l}$ be the vertices in $V_{b i g}$. For $j=1, \ldots, l$, position $S_{w_{j}}$ in the $(Z=0)$-plane such that its top left corner is at $\left(\sum_{i=1}^{j-1} 2 k\left(w_{i}\right), 2\right)$. So all these rectangles attach to the $X$-axis and abut each other.

5. Add extra vertices of degree 0 such that $V_{\text {small }}$ has exactly $N^{2}$ vertices. (This step is only done to ease the description.)

6. Sort the vertices in $V_{\text {small }}$ by non-increasing degree as $v_{0}, v_{1}, v_{2}, \ldots, v_{N^{2}-1}$. For $k=0, \ldots, N^{2}-1$, if $k=i N+j$, then place the bottom left corner of $S_{v_{k}}$ at $(2(i+j) \bmod 2 N, 2 j)$.

7. For each vertex $v \in V$, remove the top two rows from $S_{v}$ and the two rightmost columns from $S_{v}$. Vertices are now disjoint; see Fig. 2,

8. Orient each edge in $E_{c u t}$ from the endpoint in $V_{b i g}$ to the other endpoint. Orient all edges in $E_{\text {small }}$ arbitrarily.

9. For each oriented edge $e=v w \in E_{\text {cut }}$, assign a $Z^{+}$-port at $S_{v}$ with even coordinates to $e$ in such a way that at most $4 \mathrm{~m} / N$ edges are assigned to any $Z^{+}$-port of any vertex in $V_{\text {big }}$.

For any edge $e \in E_{\text {cut }} \cup E_{\text {small }}$ with an endpoint $v \in V_{\text {small }}$, edge $e$ uses the unique $Z^{+}$-port with even coordinates at $S_{v}$.

10. Construct a graph $H$ with $V(H)=E_{\text {cut }} \cup E_{\text {small }}$. For oriented edges $v w, x y \in$ $V(H)$, add the edge $\{v w, x y\}$ to $E(H)$ if the ports of $v w$ at $v$ and of $x y$ at $x$ are in the same column, or if the ports of $v w$ and $w$ and of $x y$ at $y$ are in the same row.

11. Vertex colour the graph $H$ with $\Delta(H)+1$ colours, and for each vertex $v \in V(H)$ coloured $i$ corresponding to an edge $v w$, set $h(v w) \leftarrow i$.

12. For each oriented edge $v w \in E_{\text {cut }} \cup E_{\text {small }}$ construct an edge route for $v w$ exactly as described in the algorithm in Sect. 3.1.

13. Construct edge routes for edges in $E_{b i g}$ by copying the 2-bend layout of the complete graph developed in [5]. More precisely, split $E_{b i g}$ into at most $\left|V_{b i g}\right|-1$ matchings such that no two edges within one matching are forced to cross, assign one $Z$-plane for each matching, and route each edge in the matching with at most 2 bends in this $Z$-plane.

14. Enlarge points/lines representing vertices into lines/rectangles by extending their sides parallel to the $Z$-axis from the minimum to the maximum $Z$ coordinate used in the drawing. Clip all segments of edges that overlap the box of an endpoint. 

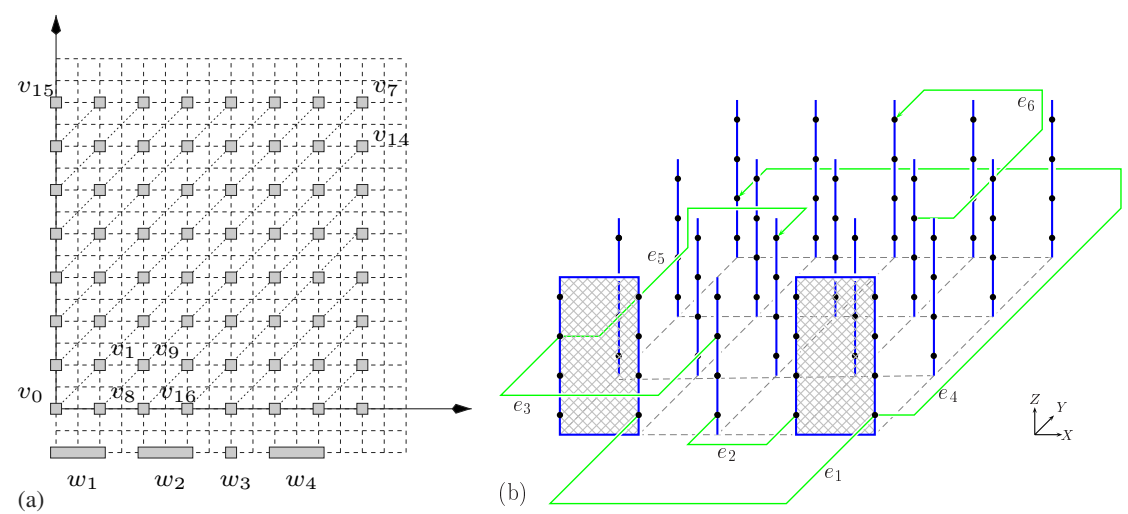

Fig. 2. (a) Vertex layout: $w_{1}, w_{2}, w_{3}, w_{4}$ belong to $V_{b i g}$. (b) Edge routes: $e_{1}, e_{2}$ and $e_{3}$ belong to $E_{b i g}, e_{4}$ and $e_{5}$ belong to $E_{c u t}$, and $e_{6}$ belongs to $E_{\text {small }}$.

Lemma 4. The maximum degree of $H$ is at most $16 m / N-2$.

Proof. (Sketch) By construction there are at most $4 \mathrm{~m} / N$ edges in $E_{\text {cut }}$ assigned to the same $Z^{+}$-port in the row with $Y$-coordinate -2 . Let a proper column be a column with even $X$-coordinate after taking away the points with negative $Y$ coordinates. Let a proper row be a row that has non-negative even $Y$-coordinate. The result holds if any proper row/column has at most $6 \mathrm{~m} / \mathrm{N}$ edges that use a $Z^{+}$-port in that row/column. This can be shown by analyzing the way vertices in $V_{\text {small }}$ have been assigned to points in proper rows/columns, and using the fact that the maximum degree of any vertex in $V_{\text {small }}$ is at most $4 \mathrm{~m} / \mathrm{N}$.

Theorem 5. The above algorithm determines an orthogonal drawing of any simple graph $G$ in $\mathcal{O}\left(m^{2} / \sqrt{n}\right)$ time, with $\mathcal{O}(m \sqrt{n})$ bounding box volume and at most four bends per edge route.

Proof. (Sketch) We start with $2 N X$-planes and $2 N+2 Y$-planes. The edges in $E_{b i g}$ may add up to $\left|V_{b i g}\right| / 2 Y$-planes, but one can show that $\left|V_{b i g}\right| \leq N / 2$.

A greedy vertex colouring of $H$ requires at most $\Delta(H)+1$ colours, so to route the edges in $E_{\text {cut }} \cup E_{\text {small }}$, we need at most $2 \cdot 16 \mathrm{~m} / N Z$-planes. To route the edges in $E_{b i g}$, we need at most $\left|V_{\text {big }}\right| \leq N / 2 Z$-planes, which is smaller by $m \geq n / 2$, so the height of the drawing above the $Z=0$ plane is at most $32 \mathrm{~m} / N$. The bounding box is therefore $2 N \times\left(\frac{9}{4} N+2\right) \times 32 m / N=144 m N+\mathcal{O}(m)=\mathcal{O}(m \sqrt{n})$.

The time-consuming stage of the algorithm is the vertex colouring of $H$, which takes $\mathcal{O}(|E(H)|)=\mathcal{O}(|V(H)| \Delta(H))=\mathcal{O}(m \cdot m / N)=\mathcal{O}\left(m^{2} / \sqrt{n}\right)$ time.

Originally there are at most six bends per edge route, and at most four bends per edge route for edges in $V_{b i g}$. During the clipping step, the first and last segment of each edge is clipped, thus every edge has at most four bends. 
The technique of Biedl and Chan [4] works for steps 10 and 11 of this algorithm as well, reducing the time complexity of our algorithm to $\mathcal{O}(m \log n)$, and decreasing the volume to $90 m \sqrt{n}+o(m \sqrt{n})$.

We can decrease the number of bends to three bends if we allow an increase in volume. In fact, the construction greatly simplifies: position the vertices as $Z$-lines in an $N \times N$-grid, assign to each edge a unique height, and route each edge as before, but omitting the middle segment. The height is then $m$, and the width and depth are both $2 N$.

Theorem 6. Every loopless graph has an orthogonal drawing, which can be computed in $\mathcal{O}(m)$ time, with $\mathcal{O}(m n)$ bounding box volume and three bends per edge route.

This algorithm is particularly appropriate for multilayer VLSI as there are no vertical edge segments ('cross-cuts'); see [1].

\section{Conclusions and Open Problems}

In this paper we have provided matching upper and lower bounds for threedimensional orthogonal box-drawings.

In particular, we showed that any algorithm to create three-dimensional orthogonal drawings that have bounded aspect ratios or are degree-restricted cannot do better than a bounding box volume of $\Omega\left(\mathrm{m}^{3 / 2}\right)$. We then gave an algorithm that matches this bound, i.e., constructs three-dimensional degreerestricted orthogonal cube-drawings with bounding box volume $\mathcal{O}\left(\mathrm{m}^{3 / 2}\right)$.

If there are no restrictions on the drawing, then we showed that no algorithm can do better than a bounding box volume of $\Omega(m \sqrt{n})$. We gave a second algorithm that matches this bound, i.e., constructs three-dimensional orthogonal drawings with bounding box volume $\mathcal{O}(m \sqrt{n})$.

The following open problems remain to be studied:

- Table 1 suggests a trade-off between the number of bends per edge and the bounding box volume. Can such a trade-off be proved? What are lower bounds for drawings where edges are allowed to have only one or two bends?

- In [5], Biedl et al. constructed drawings of $K_{n}$ with at most one bend per edge such that each vertex box has surface $2 n+4$. For any simple graph, this yields a drawing with at most one bend per edge, but this drawing is degree-restricted only if the minimum degree is $\theta(n)$. Does every graph have a three-dimensional orthogonal drawing that is both degree-restricted and has at most one bend per edge?

- Can the upper bounds on the maximum number of bends per edge in orthogonal drawings with optimal volume be improved? In particular, (a) does every graph have a 5-bend degree-restricted cube-drawing with $\mathcal{O}\left(\mathrm{m}^{3 / 2}\right)$ volume, and (b) does every graph have a 3-bend drawing with $\mathcal{O}(m \sqrt{n})$ volume? Note that $K_{n}$ does have a $\mathcal{O}\left(n^{5 / 2}\right)=\mathcal{O}(m \sqrt{n})$ volume 3 -bend drawing [5]. 


\section{References}

1. A. Aggarwal, M. Klawe, and P. Shor. Multilayer grid embeddings for VLSI. Algorithmica, 6(1):129-151, 1991.

2. N. Alon and J. Spencer. The Probabilistic Method. John Wiley \& Sons, 1992.

3. T. Biedl. Three approaches to 3D-orthogonal box-drawings. In Whitesides [17, pages $30-43$.

4. T. Biedl and T. Chan. Cross-coloring: improving the technique by Kolmogorov and Barzdin. Technical Report CS-2000-13. Department of Computer Science, University of Waterloo, Canada, 2000.

5. T. Biedl, T. Shermer, S. Whitesides, and S. Wismath. Bounds for orthogonal 3-D graph drawing. J. Graph Alg. Appl., 3(4):63-79, 1999.

6. T. Biedl, T. Thiele and D. R. Wood. Three-Dimensional Orthogonal Graph Drawing with Optimal Volume. Technical Report CS-2000-12. Department of Computer Science, University of Waterloo, Canada, 2000.

7. M. Closson, S. Gartshore, J. Johansen, and S. K. Wismath. Fully dynamic 3dimensional orthogonal graph drawing. In Kratochvíl [14, pages 49-58.

8. G. Di Battista, M. Patrignani, and F. Vargiu. A split\&push approach to 3D orthogonal drawing. In Whitesides [17, pages 87-101.

9. P. Eades, C. Stirk, and S. Whitesides. The techniques of Kolmogorov and Bardzin for three-dimensional orthogonal graph drawings. Information Processing Letters, 60(2):97-103, 1996.

10. P. Eades, A. Symvonis, and S. Whitesides. Three dimensional orthogonal graph drawing algorithms. Discrete Applied Math., 103(1-3):55-87, 2000.

11. É. K. Fogel. An elementary proof of formulae of de la Vallée Poussin (In Russian). Latvijas PSR Zinātņu Akad. Vestis, 11(40):123-130, 1950.

12. K. Hagihara, N. Tokura, and N. Suzuki. Graph embedding on a three-dimensional model. Systems-Comput.-Controls, 14(6):58-66, 1983.

13. D. Kleitman and M. Krieger. An optimal bound for two dimensional bin packing. In 16th Annual Symposium on Foundations of Computer Science (FOCS'75), pages 163-168. IEEE, 1975.

14. J. Kratochvil, editor. Symposium on Graph Drawing 99, volume 1731 of Lecture Notes in Computer Science. Springer-Verlag, 1999.

15. A. Lubotzky, R. Phillips, and P. Sarnak. Ramanujan graphs. Combinatorica, 8:261-277, 1988.

16. A. Papakostas and I. Tollis. Incremental orthogonal graph drawing in three dimensions. J. Graph Alg. Appl., 3(4):81-115, 1999.

17. S. Whitesides, editor. Symposium on Graph Drawing 98, volume 1547 of Lecture Notes in Computer Science. Springer-Verlag, 1998.

18. D. R. Wood. An algorithm for three-dimensional orthogonal graph drawing. In Whitesides [17, pages 332-346.

19. D. R. Wood. Multi-dimensional orthogonal graph drawing with small boxes. In Kratochvíl [14], pages 311-322.

20. D. R. Wood. A new algorithm and open problems in three-dimensional orthogonal graph drawing. In R. Raman and J. Simpson, editors, Proc. Australasian Workshop on Combinatorial Algorithms (AWOCA'99), pages 157-167. Curtin University of Technology, Perth, 1999. 